\title{
Acute and Chronic Stress Effects on Open Field Activity in the Rat: Implications for a Model of Depression
}

\author{
R. J. KATZ,' K. A. ROTH ${ }^{2}$ AND B. J. CARROLL \\ Mental Health Research Institute, Department of Psychiatry \\ University of Michigan Medical Center, Ann Arbor, MI 48109
}

Received 15 February 1980

\begin{abstract}
KATZ, R. J., K. A. ROTH AND B. J. CARROLL. Acute and chronic stress effects on open field activity in the rat: Implications for a model of depression. NEUROSCI. BIOBEHAV. REV. 5(2) 247-251, 1981. -The initial activity of a rat placed in novel surroundings (i.e., open field activity) has been taken as an indicator of its emotional state. We have investigated the effects of immediately antecedent stress upon open field activity in comparison with basal (i.e., unstressed) activity, and additionally, the effects of a history of chronic stress upon the above behavioral patterns. Acute exposure to a non-traumatic, non-debilitating stress (noise and light) consistently increased activity in comparison with basal activity. A history of chronic stress on the other hand reduced basal activity from control levels, and eliminated the activation response to acute stress. This lack of acute activation may bear some resemblance to depression on several grounds. Behaviorally it represents a "refractory loss of interest." Also, chronically stressed rats showed endocrine changes similar to those seen in human depressives. Finally, antidepressant treatment with the monoamine oxidase inhibitor pargyline restored the ability of chronically stressed rats to respond actively to stress.
\end{abstract}

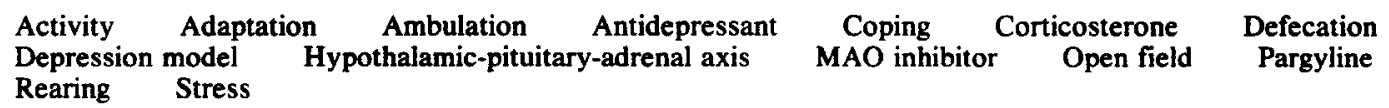

THE purpose of the present study was to demonstrate the utility of the open field test, a simple procedure used in psychological laboratories for some five decades, in the modelling of depression in the rat. Previous studies have indicated that the initial open field activity of naive subjects may be ordered along a continuum of behavioral engagement. "Emotional" rats, so termed, [11, 22, 25] show high defecation, little motor activity and low levels of grooming and rearing. They avoid the center of the field. Their engagement in their immediate surroundings may be itermed passive and limited. "Non-emotional" animals $[11,22,25]$ on the other hand move throughout the novel environment including both its center and periphery. They most typically show high levels of rearing and grooming, and limited defecation. Thus, they respond to the open field with continuous activity. We have developed simple procedures involving acute noise pre-exposure which allow manipulation of initial activity [22]. Rats given $1 \mathrm{hr}$ pre-exposure to $95 \mathrm{~dB}$ of white noise prior to initial placement in the open field showed a highly "engaged" and active behavioral profile while control cage mates, which did not receive stress, typically were "emotional", showing freezing and defecation.
Depression at a clinical level often involves a pathology of the engagement process. Klein speaks of a "profound lack of interest and investment in the environment" [16] and this is echoed in the descriptions provided by others e.g., "he [the depressive] retires from usual social activities, avoids his friends in the street ... seems bored and inattentive ... [20]." Thus, engagement of both a social and nonsocial nature is typically disrupted in clinical depression. Although identical processes might be difficult to model directly in rats, an analogous loss of engagement to novelty might also be present in the open field. A prior history of stress is believed to be a possible precipitant of certain forms of depression in humans $[1,12,14]$ and also may intensify a preexisting depression. We therefore examined whether a similar process i.e., chronic exposure to antecedent stress, could blunt the normal stress elicited activation response of the rat in an open field. To further examine possible relations to clinical depression we administered an antidepressant drug to chronically stressed rats to see if this treatment would affect responses to chronic stress.

Finally, in order to establish the physiological effectiveness of the stress procedure, to examine the endocrine con-

${ }^{1}$ Correspondence to Dr. Katz at Department of Psychology, Johns Hopkins University, Charles and 34th Street, Baltimore, MD 21218.

${ }^{2}$ Kevin Roth is currently at the Pritzker Laboratory of Behavioral Neurochemistry, Stanford University School of Medicine, Palo Alto, CA. 
comitants of normal and disrupted open field performance, and to identify endocrine changes which parallel the prominent endocrine abnormalities found in human depression [6-10] we studied the hypothalamic-pituitary-adrenal (HPA) activity of the rats through the examination of circulating steroid levels.

\section{METHOD}

Subjects. A total of $\mathbf{8 0}$ adult male Sprague-Dawley rats ( $n=10$ /cell; Charles River Farms, Portage, MI), aged 70 days at the start of testing, were housed two/cage in standard rack-mounted $25 \times 18 \times 17 \mathrm{~cm}$ stainless steel cages with food (Teklad 4.0\% fat rodent diet S-0836; Madison, WI) and tap water continuously available. Normal day/night cycles of 12 $\mathrm{hr} / 12 \mathrm{hr}$ (lights on $=0700-1900 \mathrm{hr}$ ) were programmed automatically. Rats were housed as described at least four weeks prior to testing.

Apparatus. Testing was carried out in a square white Plexiglas open field, each side of which was $1.22 \mathrm{~m}$ and the height of which was $45 \mathrm{~cm}$. The apparatus floor was divided into 16 equal squares for assessing locomotion. The field was cleaned thoroughly between tests.

Behavioral procedure. Open field testing utilized a com. plete $2 \times 2 \times 2$ factorial design in which the first factor was the presence or absence of an immediately antecedent noise stress. The acute stress manipulation was varied across conditions of chronic stress involving a basal condition (with no additional manipulation i.e., no prior stress) and a chronic stress procedure involving exposure to a series of acute stressors over a three week interval. Finally, half the rats in each of the above conditions were given daily treatment with vehicle or the monoamine oxidase inhibitor pargyline (pargyline $\mathrm{HCl}$; Sigma $\mathrm{P}-8103 ; 5 \mathrm{mg} / \mathrm{kg}$ ) during the stress period. Drug or $0.9 \%$ sodium chloride vehicle was injected once per day on a daily basis for the entire 21 day course of stress administration. Drug treatment was concomitant with chronic stress to allow a sufficient duration of chronic drug exposure, and to insure that the effects of stress upon behavior would not dissipate during drug treatment. The final drug injection occurred 48-72 hours prior to behavioral testing.

\section{Description of First Factor}

Acute stress procedure. During the week immediately preceding testing laboratory personnel entered the housing facility at the time when testing was later planned, and performed routine tasks. Subjects were therefore accustomed to the later presence of an experimenter. All testing was carried out after the onset of the dark cycle, between 2100 and 2300 $\mathrm{hr}$. On the day of stress both subjects in a cage were simultaneously removed and placed in $48 \times 27 \times 20 \mathrm{~cm}$ polypropylene cages (Scientific Products) for individual transport. The first rat (chosen randomly) was immediately taken $6 \mathrm{~m}$ down a darkened corridor to the test room. Time for transport and placement in the apparatus was less than $30 \mathrm{sec}$. The remaining subject was transported a similar distance to a brightly lit room $(8 \times 70 \mathrm{~W}$ lamps). The cage was placed $1 \mathrm{~m}$ from a speaker emitting $95 \mathrm{~dB}$ as white noise. After a $1 \mathrm{hr}$ exposure to the noise-light stress the experimental animal was transported and tested in the open field as described below.

Testing procedure. For all subjects the test room was illuminated by six GE F 96712R fluorescent lights which emitted dim (500 mphot) red light $(600-700 \mathrm{~nm})$ that was subliminal for the subjects but allowed experimental observation. A background noise of $40-50 \mathrm{~dB}$ was provided by the air circulation system. Subjects were placed in a corner of the open field facing the apparatus wall, and a six minute observation period was automatically timed. The six minute interval was utilized based upon preliminary observations from our laboratory, and in order to allow comparison with previous studies. For two measures this was further divided into two three-minute periods, with the first 3 minute block taken as the primary datum. Rationale for shorter intervals may be found in previous papers (e.g., $[22,25])$. To allow within-experiment comparisons, 6 minute data also are presented. Again, this was to allow a more adequate comparison. The following categories of behavior were recorded; defecation score (boluses/session), latency to initial defecation (in sec), latency to leave home square (in sec), outside squares traversed (in 3 and $6 \mathrm{~min}$ ), center field penetration (i.e., total inside squares entered in $6 \mathrm{~min}$ ), and rearing activity (in 3 and $6 \mathrm{~min}$ ). At the close of testing subjects were removed, immediately (less than $30 \mathrm{sec}$ ) sacrificed by decapitation, and trunk blood collected in heparinized tubes. Following centrifugation at $2400 \mathrm{rpm}$ for $30 \mathrm{~min}$, plasma was collected and frozen at $-40^{\circ}$ for later corticosterone determination, using the method of Murphy [20].

\section{Description of Second Factor}

Chronic stress regimen. The stress procedure was designed to maximize the unpredictable nature of both the stimulus properties of stress and time of stress delivery. Stressors were administered separated from each other by one to two days over a total period of 21 days and were administered throughout the circadian cycle between 0800 and $2100 \mathrm{hr}$. The following stressors were used: switching cagemates ( 3 times); $24 \mathrm{hr}$ isolation in which rats were removed from normal double housing to individual cages for 24 hr prior to being returned to double housing (1 time); 30 minutes of scrambled unpredictable footshock ( 3 times; approximately one $1.0 \mathrm{~mA}$ shock $/ 60 \mathrm{sec}$; shocks averaged 10 $\mathrm{sec}$ in duration and ranged from 1 to $15 \mathrm{sec}$; the shock generator was a Lafayette Instruments A-615 C master shocker which utilized a neon-grid principle of scrambling); $46 \mathrm{hr}$ food deprivation ( 2 times); $46 \mathrm{hr}$ water deprivation ( 2 times): 5 minutes swim in $4.0^{\circ} \mathrm{C}$ ice-water with temperature maintained by the addition of ice as necessary ( 3 times); 30 and 60 minutes of horizontal shaker stress at 160 displacements/min using an Eberback laboratory shaker; tail pinch from a rubber dam forceps applied $1 \mathrm{~cm}$ from the base of the tail, forceps were closed to the first notch and pressure was maintained for $1 \mathrm{~min}$ ( 2 times). Further details of the tail pinch procedure may be found in [14]. The precise order of stress administration is presented in Table 1. Testing occurred $48-72 \mathrm{hr}$ following the final stress. Handling controls were included.

Statistical Analysis. All data are presented as means and standard errors. The data were analyzed using univariate random design analyses of variance. Further post hoc comparisons were by Duncan's multiple range test.

\section{RESULTS}

Acute stress increased a number of motor behaviors and decreased defecation in comparison with controls. Chronic stress generally lowered open field activity and prevented the acute activation effect, and this was restored by chronic antidepressant treatment. Drug treatment did not in itself produce any remarkable changes upon normal or stress elicited activity. 
TABLE 1

OPEN FIELD BEHAVIORS IN THE RAT (MEAN \pm S.E.M.; $n=10 /$ CELL)

\begin{tabular}{|c|c|c|c|c|c|c|c|c|}
\hline \multirow[t]{2}{*}{ Category } & \multicolumn{2}{|c|}{ Vehicle } & \multicolumn{2}{|c|}{$\begin{array}{l}\text { Vehicle-Chronic } \\
\text { Stress }\end{array}$} & \multicolumn{2}{|c|}{ Pargyline } & \multicolumn{2}{|c|}{$\begin{array}{l}\text { Pargyline-Chronic } \\
\text { Stress }\end{array}$} \\
\hline & Basal & Acute & Basal & Acute & Basal & Acute & Basal & Acute \\
\hline $\begin{array}{l}\text { Outside Squares } \\
(0-3)\end{array}$ & $21 \pm 4$ & $49 \pm 5$ & $18 \pm 7$ & $24 \pm 3$ & $25 \pm 3$ & $44 \pm 6$ & $20 \pm 7$ & $33 \pm 3$ \\
\hline $\begin{array}{l}\text { Outside Squares } \\
(0-6)\end{array}$ & $59 \pm 2$ & $71 \pm 6$ & $28 \pm 6$ & $19 \pm 4$ & $67 \pm 6$ & $75 \pm 4$ & $33 \pm 7$ & $49 \pm 4$ \\
\hline Latency (sec) & $10 \pm 3$ & $5 \pm 1$ & $29 \pm 7$ & $33 \pm 5$ & $15 \pm 4$ & $6 \pm 1$ & $30 \pm 9$ & $11 \pm 3$ \\
\hline $\begin{array}{l}\text { Inside Squares } \\
\quad(0-6)\end{array}$ & $3 \pm 1$ & $4 \pm 1$ & $0 \pm 0$ & $1 \pm 1$ & $1 \pm 1$ & $4 \pm 2$ & $2 \pm 1$ & $5=3$ \\
\hline $\begin{array}{l}\text { Rearing } \\
\quad(0-3)\end{array}$ & $17 \pm 2$ & $23 \pm 1$ & $14 \pm 2$ & $18 \pm 3$ & $16 \pm 5$ & $21 \pm 4$ & $13 \pm 4$ & $22 \pm 3$ \\
\hline $\begin{array}{l}\text { Rearing } \\
\qquad(0-6)\end{array}$ & $47 \pm 5$ & $45 \pm 4$ & $27 \pm 5$ & $18 \pm 5$ & $51 \pm 6$ & $49 \pm 4$ & $37 \pm 6$ & $41 \pm 3$ \\
\hline $\begin{array}{l}\text { Defecation } \\
\quad(0-6)\end{array}$ & $3 \pm 1$ & $1 \pm 0$ & $5 \pm 1$ & $4 \pm 1$ & $3 \pm 1$ & $1 \pm 1$ & $7 \pm 1$ & $2 \pm 1$ \\
\hline $\begin{array}{l}\text { Defecation } \\
\text { Latency (sec) }\end{array}$ & $33 \pm 4$ & $45 \pm 6$ & $12 \pm 3$ & $23 \pm 3$ & $35 \pm 5$ & $32 \pm 5$ & $24 \pm 4$ & $40 \pm 5$ \\
\hline $\begin{array}{l}\text { Corticosterone } \\
(\mu \mathrm{g} / \mathrm{dl})\end{array}$ & $22 \pm 2$ & $43 \pm 8$ & $34 \pm 3$ & $77 \pm 9$ & $25 \pm 3$ & $69 \pm 6$ & $18 \pm 3$ & $49 \pm 12$ \\
\hline
\end{tabular}

These findings were confirmed statistically, and are presented in Table 2 . It may be seen that for initial outside squares activity there are clear and significant differences across categories, $\mathrm{F}(7,72)=4.1, p<0.01$. Of particular interest are the following significant differences across cells: stress elevated initial activity in comparison to an unstressed control; chronic stress reduced basal activity and the acute response. Drug treatment although not in itself behaviorally effective restored the response to an acute stress. This was further confirmed by a similar significant pattern for the 6 minute block, $\mathrm{F}(7,72)=8.1, p<0.001$, and by other measures as well. For example, it may be seen that latency scores for initial movement reflected an overall change, $F(7,72)=7.1$, $p<0.001$. Again the acute stress group was more active in initiating motor activity than its respective control. Initial activity latency was significantly prolonged by prior chronic stress, however. This was partially but significantly reduced by drug treatment. Center field penetration followed an essentially similar course. However, the differences between groups did not occur at a level of acceptable statistical significance, $F(7,72)=1.3$, N.S. This lack of overall significance precluded further statistical analysis. It might be noted, however, that although effects upon center field penetration were not established beyond chance levels the direction of effects showed a striking ordinal similarity to the previous measures.

Effects of the various manipulations upon initial (i.e., $0-3$ min) rearing activity indicated an overall between groups effect which was significant, $F(7,72)=2.9, p<0.01$. Post-hoc comparisons indicated a significant increase in activity due to acute stress; a reduction in rearing and elimination of the acute stress effect in chronically stressed rats; and a significant restoration of the acute stress response in the drug treated group. It should be noted that although an overall difference was present for the $0-6$ minute measure as well, $\mathrm{F}(7,72)=4.0, p<0.001$, no significant elevation due to stress
TABLE 2

\begin{tabular}{ll}
\hline Day & \multicolumn{1}{c}{ Treatment } \\
\hline 1 & Shock \\
2 & Food deprivation (46 hr) \\
3 & Cold swim \\
4 & Water deprivation (46 hr) \\
5 & \\
6 & Tail pinch \\
7 & Shaker stress \\
8 & \\
9 & Cold swim \\
10 & Shock \\
11 & Switch cage mates \\
12 & Tail pinch and switch cage mates \\
13 & Remove food (46 hr) and increase housing density (4/cage) \\
14 & Isolation housing \\
15 & Switch cage mates \\
16 & Cold swim \\
17 & \\
18 & Shock \\
19 & Water deprivation (46 hr) \\
20 & Shaker \\
$22-23$ & Test \\
\hline
\end{tabular}

was present. This reflects a decline in the overall rearing level of stressed rats in the final block.

Defecation scores followed a pattern essentially converse to those seen for motor activity. There was an overall groups effect, $\mathrm{F}(7,72)=6.0, p<0.001$. As might be predicted, acute stress reduced defecation and chronic stress increased defecation. In addition, drug treated animals showed a significant 
reduction in defecation scores. This may be seen to reflect in part an elevated basal condition. Consistent with previous observations a significantly greater reduction occurred to stress in drug-treated compared with chronically stressed groups. A further measure of defecation (latency) followed a converse course. There was an overall significant groups effect, $F(7,72)=5.1, p<0.001$. The increase in defecation latency produced by stress was significant, but was present also after chronic stress.

Plasma corticosterone data are presented in the same table. There was an overall effect of groups, $F(7,72)=9.1$, $p<0.001$. Of particular interest were significant elevations in plasma corticosterone levels in acutely stressed subjects across conditions. The chronic stress procedure was effective in significantly elevating the basal corticosterone levels of control subjects in comparison with subjects not receiving chronic stress. An additional significant lowering of corticosterone was brought about by drug treatment.

\section{DISCUSSION}

The plasma corticosterone levels may be taken as an external marker of central nervous system, particularly limbic-hypothalamic, pathophysiology since control of the HPA axis is based upon long and short feedback loops operating in part through the limbic system. It has been argued that disruptions of plasma cortisol which are found in clinical depressions are results of such changes [6-10].

It is apparent in the present results that chronic stress resulted in an elevation in the major circulating corticosteroid of the rat, and this paralleled disrupted behavioral activation. At least one previous report suggested a similar tonic endocrine elevation and subsequent behavioral disruption with chronic stress [21]. It may be speculated that analogous mechanisms are involved in both reports, and that both may bear a relation to clinical findings.

The results upon HPA activity following pargyline treatment merit some additional comment. Pituitary-adrenal normalization occurred in conjunction with a restoration of normal open field performance. Changes in HPA pathology also closely track behavioral improvement in human depression [8]. Therefore the present findings with pargyline also appear to possess some characteristics resembling the normal course of clinical improvement with pharmacotherapy. The significance of partial and complete behavioral remission is commented upon in greater detail below.

Pretreatment of rats with a series of unpredictable stressors had two related effects upon post-stress open field behavior. In comparison with unstressed subjects normal open field activity (e.g., outside squares, center field penetration, rearing) was moderately depressed and furthermore the ability to respond to an acute and normally activating stimulus was reduced significantly. It should be emphasized that most models of depression employing chronic stress utilize a single stress modality. Differential patterns of accomodation and adaptation may be expected depending upon stress predictability as to type and onset.

Several explanations of the behavioral effects of chronic stress may be advanced. The first explanation rests upon a cognitive principle as advanced by Seligman and co-workers (e.g., $[17,23])$, and suggests some fundamental alteration in cognition, i.e., a "failure to cope" representing a learned association between the organism's actions on the one hand, and an absence of predictable environment outcome on the other was brought about by the chronic stress regimen. This cognitive alteration presumably was translated in the present circumstances to a failure to become active to a novel stress. It must be noted that cognitions are of necessity inferential, and that there is no a priori reason why a cognitive deficit should lead to a failure of behavioral activation, as opposed to any other behavioral response. Thus, although the findings may in principle be accounted for by a helplessness explanation, they are so accounted in a post-hoc fashion.

A second explanation advanced by Anisman and coworkers (e.g., $[1,2])$ and Weiss and co-workers (e.g. , [12.27]) suggests the importance of neurochemical depletion in producing a direct loss of motor activity. Indeed it is clear from their earlier findings that in many cases the catecholamine depleting effects of stress are sufficient to account for apparent instances of learned helplessness. It should be noted that neurochemical adaptation may occur to a single type of stressor upon repeated presentation [12]. We again emphasize that a multiplicity of stress types was utilized in the present design.

Given the effectiveness of antidepressant drugs known to reverse specific deficits of the catecholamines presumed to be involved in reduced activity, explanations advanced by Anisman, Weiss and others must be seriously considered. Indeed an explanation based upon a motor activation deficit leads to predictions quite similar to the observed findings. It must again be noted, however, that stressors may have very different, indeed opposing, effects upon amine levels depending upon their type and duration [24].

It is not our intention to advance the motor deficit hypothesis as the sole or exclusive explanation of the present results. Indeed in closing we would point to Burchfield's recent attempt at a novel synthesis of the stress literature as a potentially useful further explanation. Burchfield [5] acknowledges the influence of both cognitive and physiological factors in stress adaptation (although emphasizing the former) and draws particular attention to the unique properties of chronic intermittent stress exposure. This last theory emphasizes the importance of conditioned endocrine responses which may, in turn affect stress related arousal. In particular, conditioned responses may facilitate adaptation to particular stressors. In the present experiment chronic stress reduced final activation to an acute stress.

Although her model is more suited to the intermittent presentation of a single stress it may be possible to extend her approach to include exposure to a variety of stressors, particularly as we outline above. Our approach emphasizes the joint influence of chronicity and unpredictability in producing behavioral alterations. Future papers in this series will further clarify the physiological and pharmacological specificity of the chronic stress effect as a possible depression model.

\section{REFERENCES}

1. Anisman, H., L. Grimmer, J. Irwin, G. Remington and L. S. Sklar. Escape performance after inescapable shock in selectively bred lines of mice. Response maintenance and catecholamine activity. J. comp. physiol. Psychol. 93: 229-242, 1979.
2. Anisman, H., G. Remington and L. S. Sklar. Effect of inescapable shock on subsequent escape performance catecholaminergic and cholinergic mediation of response initiation and maintenance. Psychopharmacology 61: 107-124, 1979. 
3. Blackwell, R. E. and R. Guillemin. Hypothalamic control of adrenohypophyseal secretions. A. Rev. Physiol. 35: 357-390, 1973.

4. Bruning, J. L. and B. L. Kintz. Computational Handbook of Statistics, (2nd ed.). Glenview Illinois: Scott Foresman and Co., 1977.

5. Burchfield, S. R. The stress response, a new perspective. Psychos. Med. 41: 661-672, 1979.

6. Carroll, B. J. Psychiatric disorders and steroids. In: Neuroregulators and Psychiatric Disorders, edited by E. Usdin and D. Hamburg. New York: Oxford University Press, 1977.

7. Carroll, B. J., G. C. Curtis and J. Mendels. Neuroendocrine regulation in depression. I. Limbic system-adrenal cortical dysfunction. Archs gen. Psychiatry 33: 1039-1044, 1976.

8. Carroll, B. J., G. C. Curtis and J. Mendels. Neuroendocrine regulation in depression. II. Discrimination of depressed from non depressed patients. Archs Gen. Psychiatr 33: 1051-1058, 1976.

9. Carroll, B. J. and J. Mendels. Neuroendocrine regulation in affective disorders. In: Hormones, Behavior and Psychopathology, edited by E. J. Sachar. New York: Raven, 1976.

10. Carroll, B. J. The hypothalamus pituitary adrenal axis in depression. In: Handbook of Studies on Depression, edited by G. D. Burrows. Amsterdam: Excerpta Medica, 1977.

11. Denenberg, V. H. Open field behavior in the rat-what does it mean? Ann. N. Y. Acad. Sci. 159: 852-859, 1969.

12. Glazer, H. I. and J. M. Weiss. Long term interference effect; an alternative to learned helplessness. J. exp. Psychol. Anim. Behav. Proc. 2: 202-213, 1976.

13. Hatotani, N., J. Nomura, T. Yamaguchi and I. Kitayama. Clinical and experimental studies on the pathogenesis of depression. Psychoneuroendocrinology 2: 115-130, 1977.

14. Katz, R. J. and K. A. Roth. Tail pinch induced stress arousal facilitates brain stimulation reward. Physiol. Behav. 22: 193194, 1979.
15. Klein, D. Endogenomorphic depression: A conceptual and terminological revision. Archs gen. psvichiat. 37: 447-454. 1974.

16. Leff, M. J., J. F. Roatch and W. E. Bunney. Environmental factors preceding the onset of severe depressions. Psychiatry 33: $293-311,1970$.

17. Maier, S. F. and M. E. P. Seligman. Learned helplessness: theory and evidence. J. exp. Psychol. (Gen) 105: 3-46, 1976.

18. Mayer-Gross, W., E. Slater and M. Roth. Clinical Psychiatry. (3rd ed.). Baltimore: Williams and Wilking, 1969.

19. Murphy, B. E. P. Some studies of the protein binding of steroids and their application to the routine micro and ultramicro measurement of various steroids in body fluids by competitive protein-binding radioassay. J. clin. Endocr. 27: 973-990. 1967.

20. Pollard, I., B. M. White, J. R. Bassett and K. Cairncross. Plasma glucocorticoid elevation and desynchronization of the estrous cycle following unpredictable stress in the rat. Behav. Biol. 14: 103-108, 1975.

21. Roth, K. A. and R. J. Katz. Stress, behavioral arousal and open field activity: A reexamination of emotionality in the rat. Neurosci. Biobehav. Rev. 3: 247-263, 1979.

22. Royce, J. R. On the construct validity of open field measures. Psychol. Bull. 84: 1098-1106, 1977.

23. Seligman, M. E. P. Helplessness. San Francisco: W. H. Freeman, 1975.

24. Stone, E. A. Stress and catecholamines. In: Catecholamines and Behavior 2: Neuropsychopharmacology, edited by A. J. Friedhoffes. New York: Plenum, 1975.

25. Walsh, R. N. and R. A. Cummins. The open field test-a critical review. Psychol. Bull. 83: 482-504, 1976.

26. Welker, W. I. Escape, exploratory and food-seeking responses of rats in a novel situation. J. comp. physiol. Psychol. 52: 106$111,1959$.

27. Weiss, J. M. and H. I. Glaser. The effects of acute exposure to stressors on subsequent avoidance behavior. Psychosom. Med. 37: 499-521, 1975 . 